\title{
Assessment of Cryptosporidium in Patients With Gastroenteritis by Modified Ziehl-Neelsen Staining Method in East Azerbaijan Province of Iran During 2018-2019
}

\author{
Alireza Javidmehr $^{1 \oplus}$ Yagoob Garedaghi $^{2^{*}(\mathbb{D}}$, Amir Babak Sioufi $^{3}$ \\ ${ }^{1}$ Department of Food and Agriculture, Sacramento, California, USA \\ ${ }^{2}$ Department of Parasitology, Tabriz Branch, Islamic Azad University, Tabriz, Iran \\ ${ }^{3}$ Department of Pathobiology, Tabriz University of Medical Sciences, Tabriz, Iran
}

\begin{abstract}
Introduction: Cryptosporidiosis is a worldwide zoonotic disease that is caused by Cryptosporidium species and leads to acute or chronic diarrhea and vomiting in patients. Due to the suitable conditions of East Azerbaijan province for parasite transmission, the present study was performed to evaluate the prevalence of Cryptosporidium infection in patients with gastroenteritis referred to hospitals and reference laboratories of East Azerbaijan province during 2018-2019.

Methods: A descriptive cross-sectional study was performed on patients with diarrhea who referred to hospitals and reference laboratories of East Azerbaijan province during 2018-2019. In this study, 180 fecal samples were collected from patients with gastroenteritis by cluster-random sampling. Stool samples were stored in $10 \%$ formalin and transferred to a parasitology laboratory and examined for parasite contamination by modified acid-fast staining (Modified Ziehl-Neelsen) method. Data were analyzed by chi-square test using SPSS.

Results: Out of 180 samples, the highest number of diarrhea samples belonged to the age group over 40 years (35.5\%) and the lowest belonged to the age group of $10-20$ years $(7.7 \%)$. Additionally, $56 \%$ of subjects were male and $44 \%$ were female. Of these patients, $57.7 \%$ lived in urban areas and $42.3 \%$ in rural areas. Cryptosporidium was observed in $1.6 \%$ ( 3 cases) of patients with gastroenteritis. There was a statistically significant relationship between Cryptosporidium infection and the age of patients with diarrhea $(P<0.05)$.

Conclusion: In the present study, a lower prevalence of cryptosporidiosis has been determined in comparison with previous studies in East Azerbaijan province. However, Cryptosporidium and other parasitic infections in the feces of patients with diarrhea referred to hospitals or reference laboratories need to be carefully diagnosed by appropriate parasitological methods. Keywords: Cryptosporidium, Modified Ziehl-Neelsen staining, Gastroenteritis patients, East Azerbaijan province, Iran
\end{abstract}

Received: June 22, 2020, Accepted: August 30, 2020, ePublished: October 14, 2020

\section{Introduction}

Today, in most countries of the world, gastroenteritis and acute diarrhea are among the most important medical conditions. According to the World Health Organization, 4.5 to 6 million deaths from diarrhea occur in Asia, Africa, and Latin America annually, with more than 10000 reported in the United States $(1,2)$. In the last two decades, several different genera of intestinal protozoa have been introduced as new human pathogens that can also cause diarrhea in people with normal immune systems in addition to people with defective immune systems $(3,4)$. One of these new pathogens is Cryptosporidium parvum, which is currently one of the four most important intestinal pathogens associated with diarrhea in humans (5). Cryptosporidiosis is a parasitic infection caused by Cryptosporidium that occurs in a wide range of vertebrates, including mammals, fish, reptiles, and birds $(6,7)$. Cryptosporidiosis is a gastrointestinal infection caused by Cryptosporidium that is associated with diarrhea. It can occur at any age group but it is more common in children under 5 years of age in developed countries (8-10).

The disease is a serious public health problem that is now widely regarded as one of the causes of acute infectious gastroenteritis which is considered to be lethal in immunocompromised individuals (11). The severity of the disease depends mostly on the immune status of the host; therefore, in immunocompromised individuals, it may be seen as short-term diarrhea that resolves spontaneously. However, in immunocompromised 
patients, especially those with AIDS, it presents as a lifethreatening, prolonged, cholera-like illness (12-14). The parasite causes disease by different mechanisms. Most studies of this disease have been performed on people with diarrhea and immunodeficiency; however, in this study, we examined seemingly asymptomatic and healthy people $(15,16)$. Due to the fact that the number of studies conducted in Iran on the prevalence of this parasite in humans is limited and most studies have been performed on people with immunodeficiency or diarrhea in the hospital area, the results of this study, along with other studies, can be helpful for future planning to control and prevent this parasitic disease.

\section{Materials and Methods}

This descriptive cross-sectional study was conducted on gastroenteritis patients referred to medical centers and reference laboratories of medical sciences during 20182019 in Tabriz, the capital of East Azerbaijan province of Iran. In this study, 180 fecal samples were collected from patients with diarrhea by cluster-random sampling method. The samples were then stored in $10 \%$ formalin and transferred to a parasitology research laboratory. Stool samples in the laboratory, after preparation by parasitological methods, were examined for the presence of cysts, trophozoites, and oocysts of parasites using concentration method and light microscopy (magnification: $\times 10$ and $\times 40$ ). In this study, after filling out a questionnaire by patients with gastroenteritis, fecal samples were examined in the Parasitology Department in terms of physical condition and consistency of the feces and their characteristics were recorded. Then, 5 to $10 \mathrm{~g}$ of feces were placed in the tubes containing $3.5 \mathrm{~mL}$ of commercial kit for parasite testing and the resulting suspension was centrifuged at $2000 \mathrm{rpm}$ for 2 minutes. The supernatant was discarded and the precipitate was expanded on the slide. After preparing the required samples, the remaining sediment was stored in the Eppendorf tube in the refrigerator at $4^{\circ} \mathrm{C}$ for later use. Smear was prepared for all samples and dried well at laboratory temperature. Then, by pouring a few drops of $96 \%$ methanol onto them, the samples were fixed on the slide. From the slides in the early stages, the sample was detected either positive or suspected. The prepared extensions were then stained with acid-fast stain. The slides were then examined for Cryptosporidium by light microscopy. In the acid-fast staining method, samples and slides are examined for Cryptosporidium using a light microscope with $\times 40$ and $\times 100$ lenses. In this method, Cryptosporidium oocysts are seen as round pink-red objects on a light green background $(17,18)$. Stained cysts are different in color depending on the age and condition of the cysts.

\section{Data Analysis}

Data were analyzed by chi-square test $(P<0.05)$ using
SPSS.

\section{Results}

In this study, which was performed during one year from 2018 to 2019, a total of 180 diarrheal fecal samples from patients with gastroenteritis referred to hospitals and reference laboratories of East Azerbaijan province were examined for Cryptosporidium infection. Out of 180 samples, the highest number of diarrhea samples belonged to the age group over 40 years $(35.5 \%)$ and the lowest belonged to the age group of $10-20$ years $(7.7 \%)$ (Table 1).

Moreover, $56 \%$ of the subjects were male and $44 \%$ were female. Of these patients, $57.7 \%$ lived in the city and $42.3 \%$ in the village. Patients were asked questions regarding their clinical symptoms, and the highest number of clinical symptoms following diarrhea included nausea $(22.1 \%)$, dehydration (18.4\%), and fever (15\%) and the lowest number of clinical symptoms belonged to abdominal pain (12\%).

The rate of Cryptosporidium infection was 1.6\% (3 cases) among patients with gastroenteritis using acidfast staining. The study of Cryptosporidium infection in patients with diarrhea by age showed a difference in infection rate at different ages in this study. The prevalence of Cryptosporidium infection in the age groups of 10-19 years, 20-29 years, and over 40 years was determined to be $0.5 \%$. There is a statistically significant relationship between Cryptosporidium infection and age (Table 2).

Additionally, the study of the relationship between Cryptosporidium infection and place of residence of patients with gastroenteritis showed that $1.1 \%$ lived in urban areas and $0.5 \%$ in rural areas. There was no statistically significant relationship between the rate of Cryptosporidium infection and place of residence (city/ village) (Table 3$)(P>0.05)$.

\section{Discussion}

Cryptosporidium is an intestinal protozoan that causes self-limiting diarrheal disease. It can cause severe disease in people with AIDS or other forms of immunodeficiency. This protozoan is the causative agent of cryptosporidiosis,

Table 1. Frequency Distribution of Patients With Diarrhea Referred to Hospital and Laboratory Centers in East Azerbaijan Province by Age Groups During 2018-2019

\begin{tabular}{lc}
\hline Patients A (year) & No. $(\%)$ \\
\hline$<5$ & $29(16.1)$ \\
$5-10$ & $16(8.8)$ \\
$10-20$ & $14(7.7)$ \\
$20-30$ & $22(12.2)$ \\
$30-40$ & $35(19.4)$ \\
$>40$ & $64(35.5)$ \\
Total & $180(100)$ \\
\hline
\end{tabular}


Table 2. Frequency Distribution of Cryptosporidium Infection in Patients With Gastroenteritis Referred to Hospital and Laboratory Centers in East Azerbaijan Province by Age During 2018-2019

\begin{tabular}{|c|c|c|c|c|c|c|c|}
\hline \multirow{3}{*}{ Parasitic infection } & \multicolumn{7}{|c|}{ Patients' Age (y) } \\
\hline & $<5$ & $5-9$ & $10-19$ & $20-29$ & $30-39$ & $>40$ & Total \\
\hline & No. $(\%)$ & No. $(\%)$ & No. $(\%)$ & No. $(\%)$ & No. $(\%)$ & No. $(\%)$ & No. $(\%)$ \\
\hline Infected & $0(0)$ & $0(0)$ & $1(0.5)$ & $1(0.5)$ & $0(0)$ & $1(0.5)$ & $3(1.6)$ \\
\hline Non-infected & $29(16.1)$ & $16(8.8)$ & $13(7.2)$ & $2(11.6)$ & $35(19.4)$ & $63(35)$ & $177(98.4)$ \\
\hline Total & $29(16.1)$ & $16(8.8)$ & $14(7.7)$ & $22(12.2)$ & $35(19.4)$ & $64(35.5)$ & $180(100)$ \\
\hline
\end{tabular}

Table 3. Frequency Distribution of Cryptosporidium Infection in Patients with Gastroenteritis in Patients Referred to Hospital and Laboratory Centers in East Azerbaijan Province by Place of Residence During 2018-2019

\begin{tabular}{lccc}
\hline \multirow{2}{*}{ Parasitic infection } & Urban & Rural & Total \\
\cline { 2 - 4 } & No. $(\%)$ & No. $(\%)$ & No. $(\%)$ \\
\hline Infected & $2(1.1)$ & $1(0.5)$ & $3(1.6)$ \\
Non-infected & $102(56.6)$ & $75(41.8)$ & $177(98.4)$ \\
Total & $104(57.7)$ & $76(42.3)$ & $180(100)$ \\
\hline
\end{tabular}

which is one of the zoonotic diseases of humans and animals. The disease manifests itself in two clinical forms: acute self-limiting gastroenteritis in immunocompromised individuals and chronic diarrhea in immunodeficiency individuals and the main transmission route of infection is oral $(19,20)$. The parasite may be transmitted directly by contact with an infected human or indirectly from a contaminated environment such as water and food (21). In this study, in 180 samples obtained from patients with gastroenteritis in the hospital and laboratory center of East Azerbaijan province, the prevalence of Cryptosporidium was $1.6 \%$. The prevalence of this parasite in recent studies in Iran in gastroenteritis patients, AIDS patients, and school children has been reported to vary from $0.1 \%$ to $7.7 \%$. The prevalence of Cryptosporidium is estimated to be between 1 and 3\% in Europe and North America, about $5 \%$ in Asia, and $10 \%$ in Africa. Protozoans are also the cause of diarrhea in 10 to $20 \%$ of people with AIDS living in industrialized countries, reaching up to $50 \%$ in developing countries (22-26). There are several reports of the prevalence of these protozoans in Iran in recent years. In a study conducted by researchers at Iran University of Medical Sciences in 1993, the rates of infection in patients with gastroenteritis, Hodgkin's disease, lymphoblastic leukemia, and renal failure were $2.97 \%, 12.5 \%, 6.75 \%$, and $4.5 \%$, respectively $(27,28)$. East Azerbaijan Province due to its traditional and industrial livestock production, as well as climatic conditions, high humidity, nutritional culture, and abundance of surface water has the potential for causing zoonotic diseases on a large scale and it seems that zoonotic parasites are of great importance in this province. As a result, the diagnosis of Cryptosporidium infection in patients with gastroenteritis is important at the level of hospital and laboratory centers in East Azerbaijan province, considering that patients refer to these centers from all over the province. Therefore, the prevalence of Cryptosporidium infection in our country is less than $5 \%$, and it is $1.6 \%$ in the present study. Since East Azerbaijan province has a temperate climate suitable for parasite survival, the low prevalence can be due to the improved health status of the study population, the number of samples, and less contact with animals, especially domestic animals. The reported results of studies in other parts of the world showed a difference in the prevalence of Cryptosporidium ranging from 3.8\% to $17 \%(29,30)$.

The number of studies conducted in the world on the prevalence of Cryptosporidium infection is somewhat higher compared to Iran (31). Probably one of the reasons for the low prevalence of Cryptosporidium in human societies of East Azerbaijan is the low level of infection in animals, which unfortunately has not been sufficiently studied. In addition, the increased level of literacy, improved public health, widespread use of treated water, and the implementation of large-scale health-related projects recommended by World Health Organization in recent years in Iran such as family physicians throughout the country at a high level could be considered the reasons for the decrease in the prevalence of parasitic infections. Therefore, the geographical realms, how to cultivate roses, livestock breeding, and most importantly the flow of streams from contaminated areas and beds following the seasonal rains are effective factors in increasing the prevalence of Cryptosporidium and other intestinal parasites in the region.

\section{Conclusion}

Identification of parasite species by parasitological and molecular methods in future studies in all treatment centers should be considered. It is also suggested that by introducing this parasite togeneraland specialist physicians and laboratory staff and holding retraining courses, as well as encouraging medical diagnostic laboratories to use modified Ziehl-Neelsen staining method, which is an easy method for the identification of this pathogen, more efforts should be made for the diagnosis of this parasite in the requested tests in patients with gastroenteritis. On the other hand, Cryptosporidium is known as an important cause of diarrhea, especially in patients with AIDS and weakened immune system. Although there is no known effective treatment for parasitic infections, especially in those with AIDS, mortality from cryptosporidiosis in 
immunocompromised patients, especially AIDS, should be studied. Since cryptosporidiosis is a common disease between humans and animals, the high prevalence of infection in animals, especially domestic ruminants, results in reduced growth, general weakness, and thinness of the animal, which is considered in terms of loss and economic importance.

\section{Conflict of Interests}

The authors declare that they have no conflict of interests.

\section{Ethical Issues}

In this research, ethical considerations have been fully observed.

\section{Acknowledgements}

We would like to express our deep gratitude to Islamic Azad University, Tabriz Branch, for offering valuable theoretical and practical contributions to the research team in the present study.

\section{Authors' Contributions}

YG did the writing of the manuscript. ABS did the editing of the manuscript. AJ did data collection and statistical analysis.

\section{Funding}

The authors received no financial support for the research, authorship, and publication of this article.

\section{References}

1. Stokdyk JP, Spencer SK, Walsh JF, et al. Cryptosporidium incidence and surface water influence of groundwater supplying public water systems in Minnesota, USA. Environ Sci Technol. 2019;53(7):3391-3398. doi:10.1021/acs.est.8b05446

2. Hunter PR, Nichols G. Epidemiology and clinical features of Cryptosporidium infection in immunocompromised patients. Clin Microbiol Rev. 2002;15(1):145-154. doi:10.1128/cmr.15.1.145-154.2002

3. Rafiei A, Rashno Z, Samarbafzadeh A, Khademvatan S. Molecular characterization of Cryptosporidium spp. isolated from immunocompromised patients and children. Jundishapur J Microbiol. 2014;7(4):e9183. doi:10.5812/jjm.9183

4. Desai NT, Sarkar R, Kang G. Cryptosporidiosis: an under-recognized public health problem. Trop Parasitol. 2012;2(2):91-98. doi:10.4103/2229-5070.105173

5. Shrivastava AK, Kumar S, Smith WA, Sahu PS. Revisiting the global problem of cryptosporidiosis and recommendations. Trop Parasitol. 2017;7(1):8-17. doi:10.4103/2229-5070.202290

6. Velásquez JN, Pantano ML, Vittar N, et al. First detection of Cryptosporidium DNA in blood and cerebrospinal fluid of HIV-infected patients. Parasitol Res. 2018;117(3):875881. doi:10.1007/s00436-018-5766-1

7. Kirkpatrick CE. Cryptosporidium infection as a cause of calf diarrhea. Vet Clin North Am Food Anim Pract. 1985;1(3):515-528. doi:10.1016/s0749-0720(15)313001

8. Smith HV, Nichols RA, Mallon $M$, et al. Natural Cryptosporidium hominis infections in Scottish cattle. Vet Rec. 2005;156(22):710-711. doi:10.1136/vr.156.22.710

9. Mi R, Wang X, Li C, et al. Prevalence and genetic characterization of Cryptosporidium in yaks in Qinghai Province of China. PLoS One. 2013;8(9):e74985. doi:10.1371/journal.pone.0074985
10. Graczyk TK, Fayer R, Cranfield MR. Zoonotic transmission of Cryptosporidium parvum: Implications for water-borne cryptosporidiosis. Parasitol Today. 1997;13(9):348-351. doi:10.1016/s0169-4758(97)01076-4

11. Fayer R, Morgan U, Upton SJ. Epidemiology of Cryptosporidium: transmission, detection and identification. Int J Parasitol. 2000;30(12-13):1305-1322. doi:10.1016/s0020-7519(00)00135-1

12. Upton SJ, Current WL. The species of Cryptosporidium (Apicomplexa: Cryptosporidiidae) infecting mammals. J Parasitol. 1985;71(5):625-629.

13. Garedaghi Yagoob, Seroprevalence of Neospora Caninum in Stray Dogs, American Journal of Animal and Veterinary Sciences. 2011; 6 (3): 100-104

14. Meinhardt PL, Casemore DP, Miller KB. Epidemiologic aspects of human cryptosporidiosis and the role of waterborne transmission. Epidemiol Rev. 1996;18(2):118-136. doi:10.1093/oxfordjournals. epirev.a017920

15. Checkley W, White AC, Jr., Jaganath D, et al. A review of the global burden, novel diagnostics, therapeutics, and vaccine targets for Cryptosporidium. Lancet Infect Dis. 2015;15(1):85-94. doi:10.1016/s1473-3099(14)70772-8

16. Ezzaty Mirhashemi M, Zintl A, Grant T, Lucy FE, Mulcahy G, De Waal T. Comparison of diagnostic techniques for the detection of Cryptosporidium oocysts in animal samples. Exp Parasitol. 2015;151-152:14-20. doi:10.1016/j.exppara.2015.01.018

17. Papini R, Bonelli F, Montagnani M, Sgorbini M. Evaluation of three commercial rapid kits to detect Cryptosporidium parvum in diarrhoeic calf stool. Italian Journal of Animal Science. 2018;17(4):1059-1064. doi:10.1080/1828051X.2018.1452055

18. Cunha FS, Peralta RHS, Peralta JM. New insights into the detection and molecular characterization of Cryptosporidium with emphasis in Brazilian studies: a review. Rev Inst Med Trop Sao Paulo. 2019;61:e28. doi:10.1590/s1678-9946201961028

19. Manser $M$, Granlund $M$, Edwards $H$, et al. Detection of Cryptosporidium and Giardia in clinical laboratories in Europe-a comparative study. Clin Microbiol Infect. 2014;20(1):O65-71. doi:10.1111/1469-0691.12297

20. Xiao L, Bern C, Limor J, et al. Identification of 5 types of Cryptosporidium parasites in children in Lima, Peru. J Infect Dis. 2001;183(3):492-497. doi:10.1086/318090

21. Weitzel T, Dittrich S, Möhl I, Adusu E, Jelinek T. Evaluation of seven commercial antigen detection tests for Giardia and Cryptosporidium in stool samples. Clin Microbiol Infect. 2006;12(7):656-659. doi:10.1111/j.1469-0691.2006.01457.x

22. Current WL, Garcia LS. Cryptosporidiosis. Clin Microbiol Rev. 1991;4(3):325-358. doi:10.1128/cmr.4.3.325

23. Boyce MR, O'Meara WP. Use of malaria RDTs in various health contexts across sub-Saharan Africa: a systematic review. BMC Public Health. 2017;17(1):470. doi:10.1186/s12889-017-4398-1

24. Bouyou-Akotet MK, Owono-Medang M, Moussavou-Boussougou

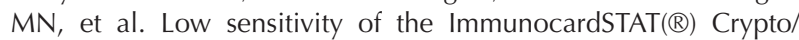
Giardia Rapid Assay test for the detection of Giardia and Cryptosporidium in fecal samples from children living in Libreville, Central Africa. J Parasit Dis. 2016;40(4):1179-1183. doi:10.1007/ s12639-015-0645-5

25. Llorente MT, Clavel A, Varea $M$, et al. Evaluation of an immunochromatographic dip-strip test for the detection of Cryptosporidium oocysts in stool specimens. Eur J Clin Microbiol Infect Dis. 2002;21(8):624-625. doi:10.1007/s10096-002-0778-1

26. Bisoffi Z, Sirima SB, Menten J, et al. Accuracy of a rapid diagnostic test on the diagnosis of malaria infection and of malaria-attributable fever during low and high transmission season in Burkina Faso. Malar J. 2010;9:192. doi:10.1186/1475-2875-9-192

27. Keshavarz A, Athari A, Haghighi A, et al. Genetic characterization of Cryptosporidium spp. among children with diarrhea in Tehran and Qazvin provinces, Iran. Iran J Parasitol. 2008;3(3):30-36.

28. Garedaghi Y, Shabestari-asl SA. Contamination rate of Toxocara spp. eggs in the public parks of Tabriz city: a short report. J Rafsanjan Univ Med Sci. 2012;11(2):173-178. [Persian]. 
29. Hamedi Y, Safa O, Haidari M. Cryptosporidium infection in diarrheic children in southeastern Iran. Pediatr Infect Dis J. 2005;24(1):86-88. doi:10.1097/01.inf.0000148932.68982.ec

30. Sharif M, Ziaee Hezarjaribi H, Gholami S. Study on prevalence rate of Cryptosporidium in patients receiving immunosuppressive drugs. J Guilan Univ Med Sci. 2004;13(51):16-22. [Persian].
31. Ghorbannia Delavar A, Nahrevanian H, Asmar M, Amirkhani A, Esfandiari B. frequency of cryptosporidiosis and isosporiasis and other enteropathogenic parasites in gastroenteritic patients (Babol and Babolsar; 2005-2006). J Babol Univ Med Sci. 2008;10(2):56-61. [Persian].

(C) 2020 The Author(s); This is an open-access article distributed under the terms of the Creative Commons Attribution License (http:// creativecommons.org/licenses/by/4.0), which permits unrestricted use, distribution, and reproduction in any medium, provided the original work is properly cited. 